\title{
Research on Mobile Context-aware Platform of Problem-Solving First
}

\author{
Wan Benting ${ }^{1}$, Kong $\mathrm{Yao}^{2}$ \\ ${ }^{1}$ Jiangxi University of Finance and Economics, Nanchang, Jiangxi, China \\ ${ }^{2}$ Jiangxi University of Finance and Economics, Nanchang, Jiangxi, China \\ ren_btw@163.com, ${ }^{b} k y y \_k o n g @ 163 . c o m$
}

\begin{abstract}
In this paper, we study the mobile situation awareness system of solving problem first, which is from the perspective of recommending resources online. The system aims at helping the team members to solve problems in as possible as short time. Experts provide materials and guidance to users via the system. The real-time scene interaction between team learners and experts makes the expert provide the accurate materials and guidance according to the needs of learners at any time. The implementation shows that the strategies illustrated in this paper improve the efficiency of solving problems.

Keywords: Mobile Context-aware; Problem-Solving; Resource-Recommending;

\section{Introduction}

The mobile situation perceptual learning has become one of the hot research subjects in the field of education, which has the perceptual advantages compared with the mobile learning. Situation perceptual learning researchers, such as Shu Lin Wang, Chia Chen Chen and Tien Chi Huang, put forward situation perception ubiquitous learning system in their study, which is based on Radio Frequency Identification (RFID) technology, wireless network, embedded handheld devices, and database technologies. Besides, it also can detect the learning behavior in the real environment, and provide learners with appropriate courseware to help them study according to the context information of learners ${ }^{[1-2] . ~ L e a r n e r s ~ d o ~ n o t ~ n e e d ~ t o ~ s p e n d ~ e x t r a ~ t i m e ~ o n ~ s e a r c h i n g ~ n e t w o r k ~}$ resources in this mobile context-aware learning. The system or experts will
\end{abstract}


provide accurate information or online tutoring for learners timely in accordance with learners' current situational information. In addition, the system or experts also instruct learners at any time by monitoring the learner's behavior ${ }^{[3]}$. In the study of Hui - Chun Chu, researchers detect the learner's behavior by the use of RFID technology. Moreover, they provide different learning guidance according to different responses to the two-tier test. The research results show that this method can not only improve the learning efficiency, but also can promote learners' learning motivation ${ }^{[4]}$.

The group collaborative learning is a kind of learning mode with planning and division through organizing learners with common learning goals. And teachers or experts are playing roles of supervisors and managers in the process of learning. The team learning online under the environment of network has gradually become one of the mainstream learning modes, mentioned in the research of Wang Xinfa, researchers of network group learning ${ }^{[5]}$.

The online collaborative learning of solving problem first based on mobile situation awareness studied in this paper combines the advantages of the situation awareness learning with team learning. It can help the team learners handle with problems as soon as possible through online learning resources recommended by experts.

\section{Mobile Context-aware Platform of Solving Problem First}

\subsection{Model of Solving Problem First}

The online collaborative learning of solving problem first based on mobile situation awareness is a kind of immediate group learning when learners meet problems need to be solved in study or life. It combines the real scene with virtual network resources and expert guidance. Expert acts as observer, supervisor and director in the collaborative learning mode, and helps learners analyze and solve problems through situational awareness learning system. Team learners interact with experts via mobile devices.

As shown in figure 1, the learning process includes two stages of the knowledge learning and problem solving activities. Learners need to learn the 
related knowledge before solving the problem. System will record the study footprints automatically and feedback them to the experts timely. Experts instruct learners according to the latest study information.

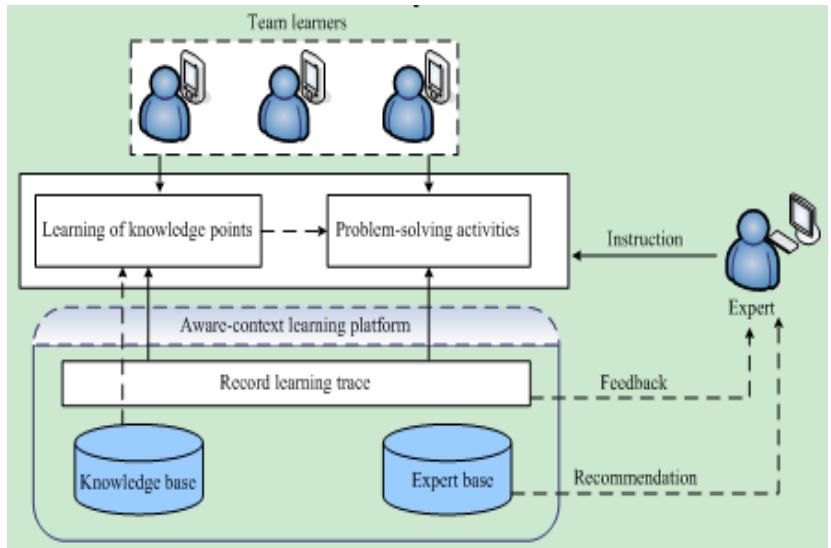

Fig.1 Online collaborative learning mode of solving problem first

\subsection{Strategies of Solving Problem First}

In this paper, we will expound the team learning strategies from the perspective of experts' recommending learning resources online. It means that the experts recommend learning content to learners according to the constructivism theory, which means that there is a certain structural sequence between knowledge points. As shown in figure 2, experts determine learning content and objectives, divide the learning content into several knowledge points, and recommend appropriate materials for each point. Team learners study the knowledge according to the materials and solve the problems by practice. Mobile context-aware learning platform automatically records learners' learning problems and learning process.

The learning of knowledge points and problem solving activities are simultaneous in the process of the team cooperative learning. For example, group member 1 learns material 1 to solve the related problems when he (or she) chooses knowledge point 1 to learn. In addition, each material recommended by experts is corresponding to a point. Learners can enter the next knowledge point to learn after fully mastering the former one. All team members respectively select one or several knowledge points to study and solve the problems, and then 
enter the group communication to help other members grasp the points.

In the process of collaborative learning online based on mobile context-aware, the learning system automatically records learning footprints and recommends appropriate experts for learners after learning problems being put forward by team learners. Moreover, the system will record students' learning progress in details and examine learning effects in learning process. At last, it sends the learning information to the experts timely so that experts master the latest information to improve the quality of instruction and team learning efficiency. Team learners can apply for experts' service online if the problems still not be solved.

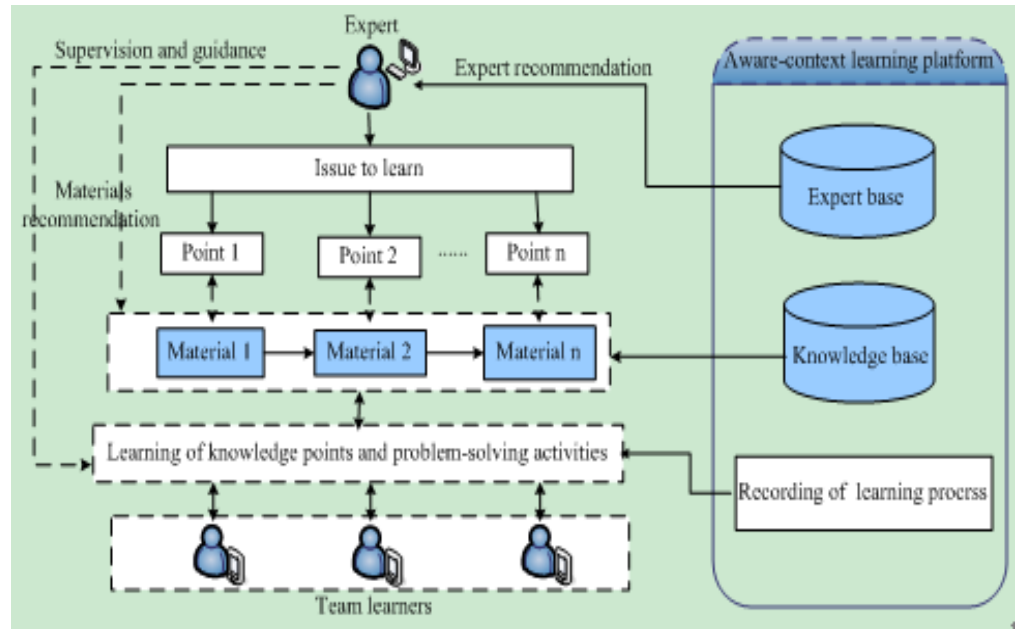

Fig. 2 Frame of online team learning process

\section{Implementation and Evaluation}

In this section, we will make the students to study the knowledge of computer host as learning tasks in order to verify the effect of the team learning based on mobile context-aware. We select 20 groups of students with the same level of knowledge and classify into two groups, experimental group and control group. The participants of 20 groups are students who are not familiar to computer. They are tutored by an outstanding student in computer major to deal with those common problems happening to computer. Each member of the experimental group is provided with a mobile learning device in an environment 
with stable network. The control groups are instructed to learn to solve problems by ways of face-to-face and hands-on teaching.

\subsection{Implementation}

We will take the problem of "desktop computers cannot be open" for example in the implementation of team learning strategies. The learning problems will be submitted to expert who makes an analysis of scenarios information to find out the causes of problems so as to define the learning content, which is mainly to handle with the problem of cpu and memory card. Learner $A$ is responsible for the relevant knowledge about the cpu failure, learner $B$ is responsible for memory card failure, and learner $C$ makes notes and interacts with experts.

As shown in Figure 3, learner $A$ is solving problem according to the courseware recommended by experts. The left side of figure 3 is the courseware about cpu failure recommended. The left on the courseware clearly shows the pictures of cpu and fan, the right on the courseware is the text about the method to solve the problem.

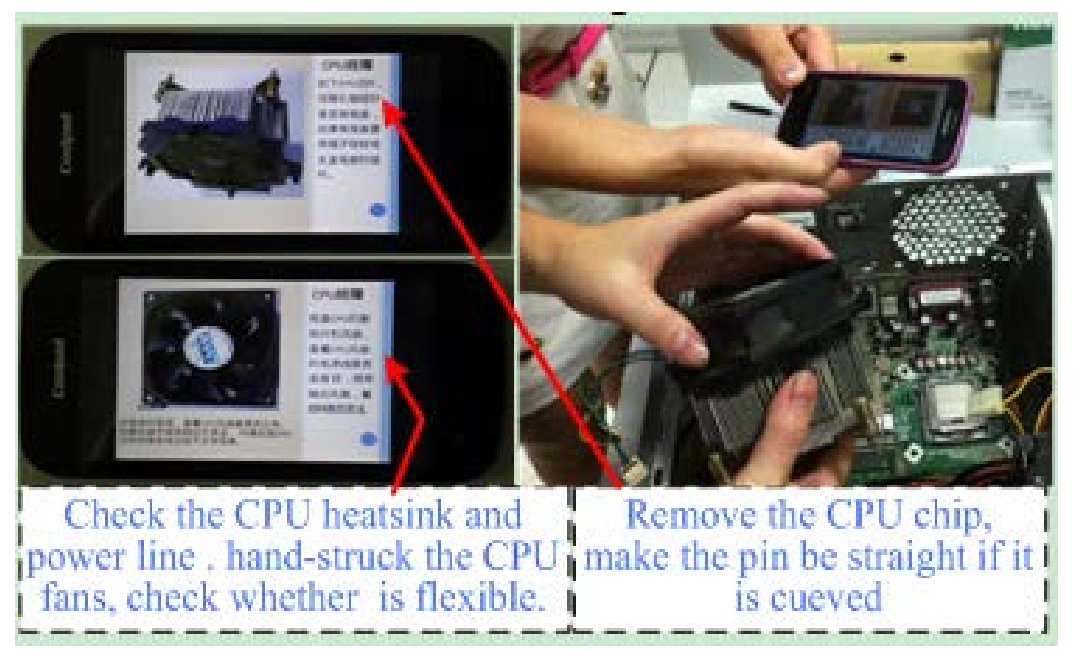

Fig. 3 Learner $A$ solves problem of cpu 


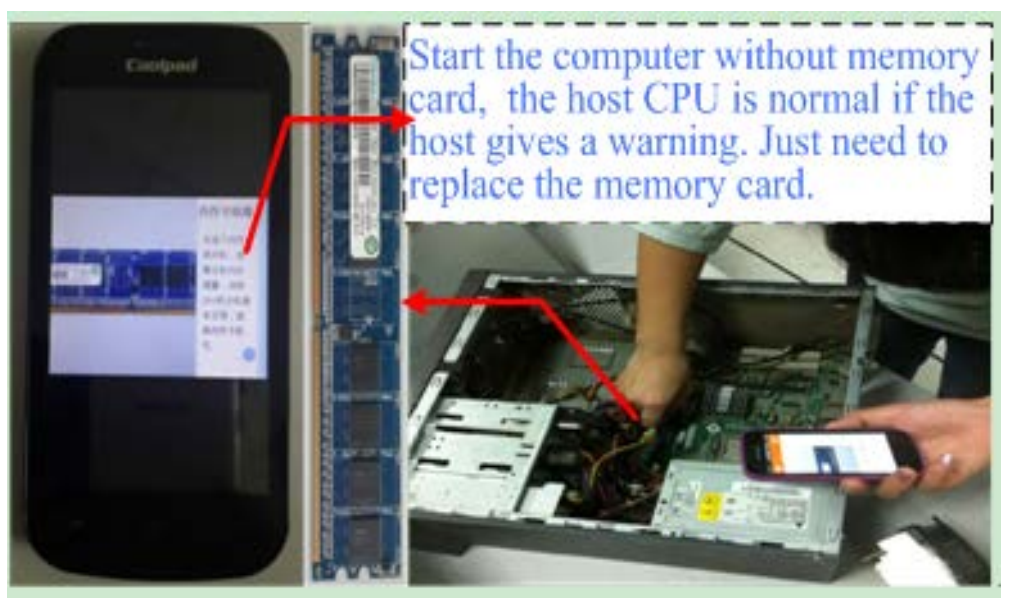

Fig. 4 Learner $B$ solves problem of memory card

In the figure 4 , learner $B$ is solving the issue of memory card failure through learning materials recommended by experts. Learner $C$ records the learning problems of other group members and learning resources recommended and gives feedbacks to the expert timely, and makes communication with experts in order to help members to solve problems better. At last the team members discuss and exchange with each other when the study is finished, so that each member grasp all the knowledge points.

\subsection{Analysis and Evaluation}

The result of the implementation shows that the learning efficiency of experimental group is close to that of control group instructed by experts in the presence. Table 1 is a schedule of experimental group and control group. The $E$ stands for experimental group, and $C$ means control group. Learning time of experimental group ranges from 19 to 25 minutes, the time of control group is from 18 to 23 minutes on premise of the problem being solved.

Table 1 Learning time of experimental group and control group

\begin{tabular}{|c|c|c|c|c|c|c|c|c|c|c|}
\hline & E1/C1 & E2/C2 & E3/C3 & E4/C4 & E5/C5 & E6/C6 & E7/C7 & E8/C8 & E9/C9 & E10/C10 \\
\hline $\begin{array}{c}\text { Learning } \\
\text { time } \\
\text { (min) }\end{array}$ & $25 / 21$ & $23 / 21$ & $20 / 19$ & $21 / 20$ & $22 / 20$ & $21 / 19$ & $19 / 18$ & $20 / 20$ & $23 / 22$ & $25 / 23$ \\
\hline
\end{tabular}


As shown in Figure 5, the mean study time of experimental group is 22.5 minutes and control group is 21 minutes. In the phase of preparation, the experimental group needs to log in the learning platform and submit learning problems, then establish a connection with expert and receive learning materials from experts, while the control group just needs to restate the learning problems to the instructor and receive learning resources. In the process of learning knowledge points, the average time of the two groups to master a point is 4.5 minutes. The differences between the two groups are mainly in the phase of preparation and problem-solving activities.

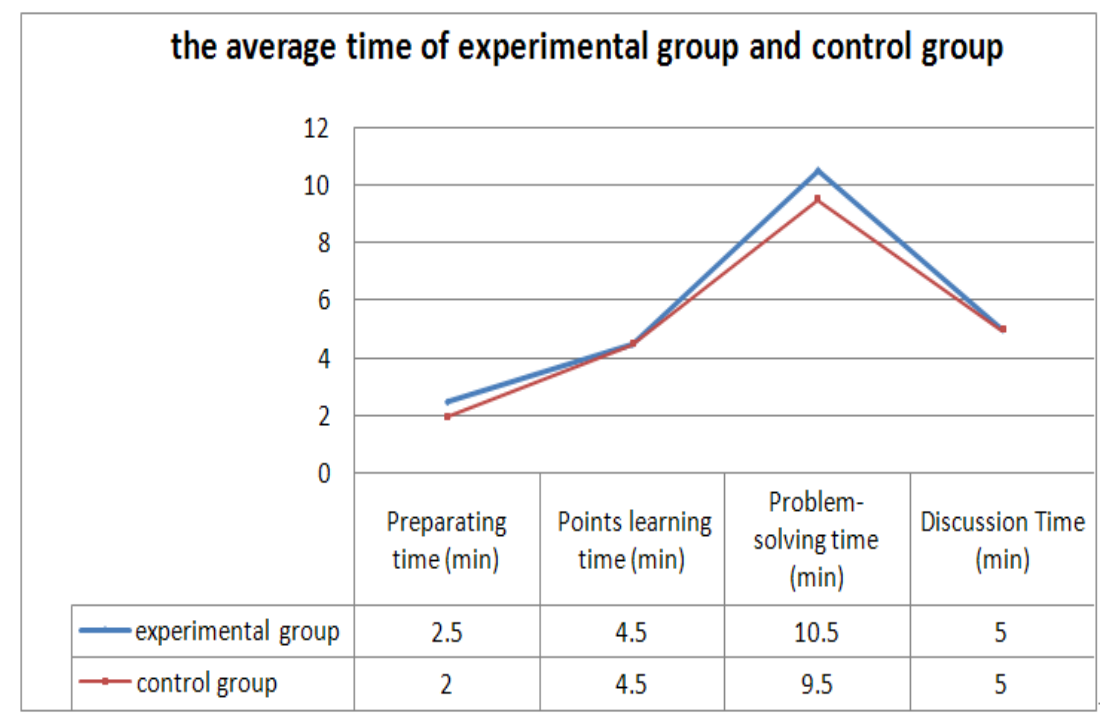

Fig. 5 Average time of experimental group and control group

\section{Conclusion}

The team learning online elaborated in this paper aims at providing learning strategies for the team learner and helping them make practical operation better in the learning process. The team handles with problems and complete tasks under the guidance of instructors. The results show that the effect of experimental group is similar to the effect of control group under the on-site guidance of experts. 


\section{Acknowledgment}

This work is supported by National Natural Science Foundation(No.61262011 and No. 61262033 and No.61262011),and Educational Office of JiangXi Province(No. KJLD12023 and No. 2010GZS0065). The authors are grateful for the anonymous reviewers who made constructive comments.

\section{Reference}

[1] Wang S L, Wu C Y. Application of context-aware and personalized recommendation to implement an adaptive ubiquitous learning system [J]. Expert Systems with applications, 2011, 38(9): 10831-10838.

[2] Chen C C, Huang T C. Learning in a u-Museum: Developing a context-aware ubiquitous learning environment [J]. Computers \& Education, 2012, 59(3): 873-883.

[3] Hwang G J, Shi Y R, Chu H C. A concept map approach to developing collaborative Mindtools for context-aware ubiquitous learning [J]. British Journal of Educational Technology, 2011, 42(5): 778-789.

[4] Chu H C, Hwang G J, Tsai C C, et al. A two-tier test approach to developing location-aware mobile learning systems for natural science courses [J]. Computers \& Education, 2010, 55(4): 1618-1627.

[5]Wang XinFa, Niu YouTian, Liu Zhen, et al. Network team learning process mode based on projects [J]. China Educational Technology, 2011, 3: 015. 